\title{
Natural Orifice Specimen Extraction: An Incisionless Approach for Colorectal Cancer (Technical Report)
}

\author{
Islam H Metwally
}

\author{
Abstract \\ Background: Natural orifice surgery represents a greatstep to the future. Difficulties arose on our current practice. Reviewing the literature \\ does not solve all the debates. \\ Report: The author suggests a simple algorithm for transanal natural orifice specimen extraction (NOSE). \\ Conclusion: Transanal extraction of colectomy and/or proctectomy specimen is a readdily feasible technique. \\ Keywords: Colon, Natural orifice, Rectum, Specimen extraction, Transanal. \\ World Journal of Laparoscopic Surgery (2018): 10.5005/jp-journals-10033-1351
}

\section{BACKGROUND}

Natural orifice specimen extraction (NOSE) for colon and rectal cancer is still taking its first steps in oncology practice. Several safety questions, as well as, technical difficulties arose with practizing this technique. ${ }^{1}$ As a part of Oncology Center Mansoura University (OCMU) center current clinical trial on natural orifice transluminal endoscopic surgery (NOTES) for colorectal cancer with ClinicalTrials. gov Identifier: NCT02549456, transanal NOSE is practiced. Different technical steps in our practice, as well as, in published series are displayed in an algorithm with videos when possible.

\section{TECHNIQUe}

Excluding cases of intersphincteric resection and some cases of ultralow anterior resection where specimen extraction is straight forward and anastomosis is done on the anal verge, the classic transanal NOSE technique is depicted in Flowchart 1 and below:

Step I: Laparoscopic sigmoidectomy, anterior, low, or ultralow resection is done.

Step II: Cut the distal end by advanced bipolar for the mesentry or mesorectum, then use scissors to cut the lumen (to avoid sealing of the wall that may hinder specimen extraction).

Step III: Either.

- A: Use a long instrument to grip the colon and retrieve it through the anal canal. Cut the proximal end and insert the anvil extracorporeal (preferred method if feasible). Here you can close the distal end with V-Loc ${ }^{\oplus}$ suture (Covidien, MA, USA) transanally or laparoscopically, so implementing a one-stapling technique (much reducing the cost) (Video 1). Otherwise, you may choose to close the stump by a linear stapler, implementing a doublestapling technique.

- B: Insert the anvil transanally, then choose.

B1: If you are planning for an end-to-end anastomosis, then cut the proximal end, insert the anvil, then take a manual purse string suture, ${ }^{1}$ or do a colotomyto insert the anvil through and use a linear stapler to close just around the anvil's tip (author's technique) (Video 2).
Department of Surgical Oncology, Oncology Center Mansoura University, Mansoura, Dakahlia, Egypt

Corresponding Author: Islam H Metwally, Department of Surgical Oncology, Oncology Center Mansoura University, Mansoura, Dakahlia, Egypt, Phone: +20 1002985865, e-mail: drislamhany@mans. edu.eg

How to cite this article: Metwally IH. Natural Orifice Specimen Extraction: An Incisionless Approach for Colorectal Cancer (Technical Report). World J Lap Surg 2018;11(3):147-148.

Source of support: Nil

Conflict of interest: None

B2: If you are planning for a side-to-end anastomosis, then do colotomy and insert the anvil with its auxiliary trocar and push against wall to make a new narrow colotomy (with/without purse string reinforcement), or use a guide tube to facilitate the anvil exteriorization. ${ }^{2}$

B3: If you are planning for a pouch reconstruction, then do the pouch with a linear stapler, then insert the anvil through the resultant opening. ${ }^{3}$

Step IV: Only applies for technique IIIB, extract the specimen either by an Endobag ${ }^{\circledR}$ (Covidien, MA, USA) (Video 3), or a camera sleeve, ${ }^{4}$ or aCai tube, ${ }^{5}$ or a rigid platform; transanal endoscopic microsurgery (TEM) (Richard Wolf, IL, USA) or transanal endoscopic operation (TEO) (Karl Storz, Tuttilingen, Germany).

Step V: Close the distal stump (as in step IIIA), but here you are mostly implementing either a double- or triple-stapling technique.

\section{ConCLUSION}

Transanal NOSE is a feasible and flexible method that can minimize the complications associated with rectal and sigmoid resection. To our opinion, any surgeon experienced in colorectal laparoscopy can easily apply this technique with a short learning curve.

\section{Supplementary Materials}

Video 1: Closing the rectal stump after transanal NOSE after low anterior resection using the direct exteriorization and

(c) The Author(s). 20180pen Access This article is distributed under the terms of the Creative Commons Attribution 4.0 International License (https://creativecommons. org/licenses/by-nc/4.0/), which permits unrestricted use, distribution, and non-commercial reproduction in any medium, provided you give appropriate credit to the original author(s) and the source, provide a link to the Creative Commons license, and indicate if changes were made. The Creative Commons Public Domain Dedication waiver (http://creativecommons.org/publicdomain/zero/1.0/) applies to the data made available in this article, unless otherwise stated. 
Flowchart 1: An algorithm summarizing steps of transanal NOSE for colorectal cancer

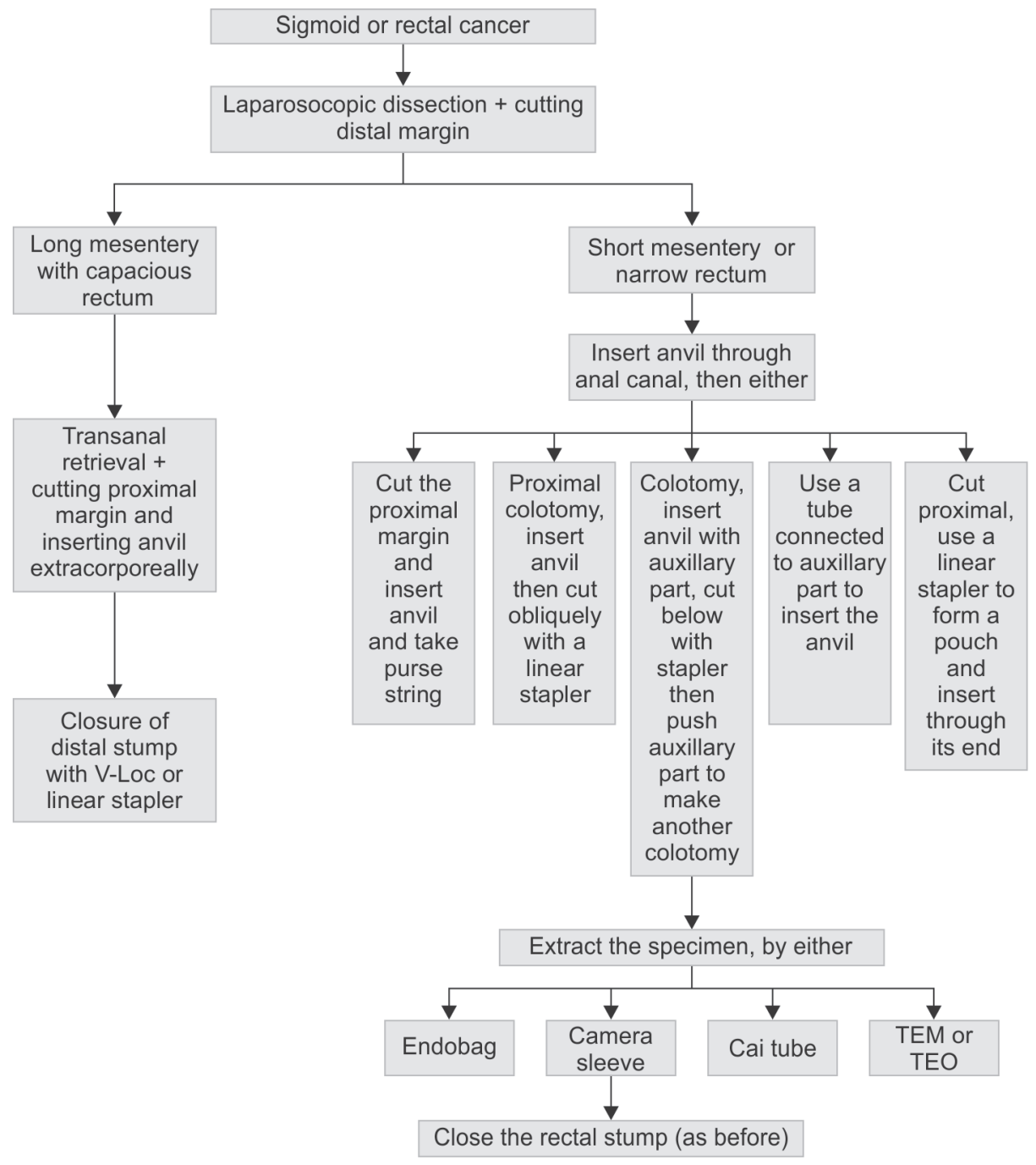

extracorporeal insertion of the anvil. https://www.dropbox.com/s/ c2morvt85od2d45/video\%201\%20M.mp4?dl=0

Video 2: Insertion of a trans-anally pushed anvil of a circular stapler into the proximal colonic stump (OCMU technique). https://www. dropbox.com/s/r95q142vil5i4w7/Video\%202\%20M.mp4?dl=0 Video 3: A second method for extraction of the specimen transanal using an endobag. https://www.dropbox.com/s/tvvkmbhj3t159x2/ Video\%203\%20M.mp4?dl=0

\section{References}

1. Lukovich $P$, Csibi N, et al. Technical questions of the transrectal specimen extraction. Magy Sebesz 2016;69(1):20-26. DOI: 10.1556/1046.69.2016.1.4.
2. Huang CC, Chen YC, et al. Totally Laparoscopic Colectomy with Intracorporeal Side-to-End Colorectal Anastomosis and Transrectal Specimen Extraction for Sigmoid and Rectal Cancers. Ann Surg Oncol 2016;23(4):1164-1168. DOI: 10.1245/s10434015-4984-3.

3. Kayaalp C, Yagci MA, et al. Laparoscopic and natural orifice transluminal restorative proctocolectomy: no abdominal incision for specimen extraction or ileostomy. Wideochirlnne Tech Maloinwazyjne 2016;11(2):115-120. DOI: 10.5114/wiitm.2016.59578.

4. Wolthuis AM, De Buck Van Overstraeten A, et al. Laparoscopic NOSE colectomy with a camera sleeve: a technique in evolution. Colorectal Dis 2015;17(5):0123-0125. DOI: 10.1111/codi.12929.

5. Cai JC, Hong XY. Laparoscopic-Assisted Natural Orifice Specimen Extraction Radical Descending Colectomy Using a Cai Tube. World J Surg 2016;40(11):2803-2807. DOI: 10.1007/s00268-016-3597-8. 